\title{
Improvement and Design of Transmitter Modifier Wind Cooling Protection for CINRAD/CB Weather Radar
}

\author{
Shi Zheng', Guan Wang2*, Xingyou Huang3,4, Chuanhai Miao1, Wanru Xing1, Simeng Chen", \\ Boshi Kang1 \\ ${ }^{1}$ Liaoning Meteorological Equipment Support Center, Shenyang, China \\ ${ }^{2}$ Liaoning Branch of Meteorological Cadre Training Institute, China Meteorological Administration, Shenyang, China \\ ${ }^{3}$ Collaborative Innovation Center for Forecast and Evaluation of Meteorological Disasters, Nanjing University of Information \\ Science \& Technology, Nanjing, China \\ ${ }^{4}$ Key Laboratory of Aerosol and Cloud Precipitation, China Meteorological Administration, Nanjing, China \\ Email: 510042894@qq.com, *carfield53222@live.cn
}

How to cite this paper: Zheng, S., Wang, G., Huang, X. Y., Miao, C. H., Xing, W. R., Chen, S. M., \& Kang, B. S. (2018). Improvement and Design of Transmitter Modifier Wind Cooling Protection for CINRAD/CB Weather Radar. Journal of Geoscience and Environment Protection, 6, 139-146.

https://doi.org/10.4236/gep.2018.611011

Received: September 20, 2018

Accepted: November 27, 2018

Published: November 30, 2018

Copyright (c) 2018 by authors and Scientific Research Publishing Inc. This work is licensed under the Creative Commons Attribution International License (CC BY 4.0).

http://creativecommons.org/licenses/by/4.0/

\begin{abstract}
Doppler weather radar has important applications in measuring the intrinsic factors of cloud, rainfall and various convective weather occurrences. Among them, CINRAD/CB Doppler weather radar is based on the requirements of the China Meteorological Administration and many units have been provided. The modulator is a critical part of the transmitter's high voltage, where high voltage, high current, and energy conversion are concentrated. It is therefore necessary to redesign the transmitter modulator cooling system protection. This article describes the new design of hardware and software solutions. The fan is a DV5214/2N DC fan from Ebmpapst, Germany. The speed is up to $5000 \mathrm{rpm}$, the power is $18.5 \mathrm{~W}$, and the single fan current is about $0.8 \mathrm{~A}$. It is powered by $28 \mathrm{~V}$. The protection board uses a DC/DC module to output a $5 \mathrm{~V}$ voltage and a $3.3 \mathrm{~V}$ voltage adjustment chip LM1117. The embedded web software is based on the TCP/IP protocol stack provided by MICROCHIP. After the cooling system is designed and installed in the radar station in Xi'an, China and other places, after long-term operation, the comprehensive test shows that the system runs well.
\end{abstract}

\section{Keywords}

Improvement and Design, Transmitter Modifier, Wind Cooling Protection, CINRAD/CB Weather Radar

\section{Introduction}

Doppler weather radar is an active remote sensing detection tool. It has impor- 
tant applications in measuring the intrinsic factors of cloud, rainfall and various convective weather occurrences (Zheng et al., 2018; Rihan \& Collier, 2010; Sayama \& Ishii, 2013). Its working principle is based on the Doppler effect and can be determined. Relative to the speed of the radar, the scatterer deducts the atmospheric wind field, the vertical velocity distribution and the turbulence under certain conditions. This is of great significance for vigilance against strong convective weather.

CINRAD/CB Doppler weather radar is based on the requirements of the China Meteorological Administration, absorbing the US WSR-88D radar design concept (Dhiram \& Wang, 2016) and advanced technology, using modern radar, microelectronics and computer technology to develop low-cost C-band full-coherence Doppler weather radar. At present, 10 units have been provided for the China Meteorological Administration, accounting for $9 \%$ of the total number of radar networks, and put into business operation or trial operation. The radar technology performance, system structure and CINRAD/CA Doppler weather radar are the same, the components are localized, and the individual technical indicators are lower than the CINRAD/CA Doppler weather radar.

The modulator is a critical part of the transmitter's high voltage, where high voltage, high current, and energy conversion are concentrated (Ahmadpoor \& Farshidi, 2012; Huang et al., 2011). The CB modulator has a charging voltage of $4000 \mathrm{~V}$ and a pulse current of up to $600 \mathrm{~A}$. The conversion of energy inevitably brings losses and efficiency problems, so the modulator generates a large amount of heat, which is mainly concentrated in the modulator soft switch-thyristor discharge circuit. Practice has proved that in the case of the modulator without air cooling, the transmitter can work for one hour to cause the modulator thyristor to be completely overheated and short-circuit damaged. The modulator's wind cooling protection circuit is critical.

Radar manufacturers have designed an improved over-cooling circuit that uses current sensing to protect it. However, due to the improper selection of the current sensor (easy magnetic saturation) and the instability of the power supply voltage, the sampling voltage is suddenly large and small, and the alarm is often false. After lowering the protection threshold, it was found that when one of the three fans stopped, it could not be protected. It is therefore necessary to redesign the transmitter modulator cooling system protection (Yeo et al., 2014; Naphon \& Wongwises, 2011; Casano \& Piva, 2015). This study redesigned the transmitter modulator cooling system protection against the above-mentioned defects of the manufacturer. The new design avoids false alarms caused by improper sensor selection and unstable power supply voltage, effectively preventing the fan from stalling and causing the device to lose protection.

\section{Overall Plan}

The CB transmitter modulator heat dissipation consists of three axial fans: two 125FZY2-S and one 100FZY2-S. Due to the continuous operation of the trans- 
mitter for a long time, the two axial fans were found to be worn out for more than 1500 hours of continuous operation, and sometimes the sound was abnormal, causing frequent damage during the flood season. Therefore, it is necessary to re-select and replace it. This time, the selected fan is the DC fan of Germany ebmpapst (model DV5214/2N). The fan has a fast speed (up to $5000 \mathrm{rpm}$ ) and a large wind flow (270, fast heat dissipation and long life).

\subsection{Design Principle}

In this wind cooling circuit, this fan has three wires because the "Ebmpapst" fan is selected (Figure 1). They are power input (two) and protection output. The fan is powered by DC voltage and the power supply range is DC $16 \mathrm{~V}-30 \mathrm{~V}$. Since the transmitter itself has a $28 \mathrm{~V}$ voltage output and the power supply is up to $600 \mathrm{~W}$, there is no power supply problem. The fan protection output adopts the OC gate open output, and its output pulse frequency is proportional to the fan speed, so this protection output can be used to monitor the actual fan speed. The fan power is $18.5 \mathrm{~W}$ and the single fan current is approximately $0.8 \mathrm{~A}$. In the meter circuit, the speed and current of the measuring fan are used to perform wind cooling protection of the transmitter modulator. Its speed and current are automatically refreshed and displayed through the web page.

\subsection{System and Circuit Structure}

The modulator cooling system mainly includes a fan cooling structure and a cooling protection circuit. The fan cooling structure mainly replaces the original modulator cooling method. The cooling protection circuit mainly includes: a microprocessor circuit, a protection detection circuit, a current sampling circuit, a network interface circuit, and a fault protection output circuit.

\section{Hardware Design}

\subsection{Power Supply}

The system uses the transmitter's own $28 \mathrm{~V}$ power supply, of which the fan is powered by $28 \mathrm{~V}$. The protection circuit board uses DC/DC module output $5 \mathrm{~V}$

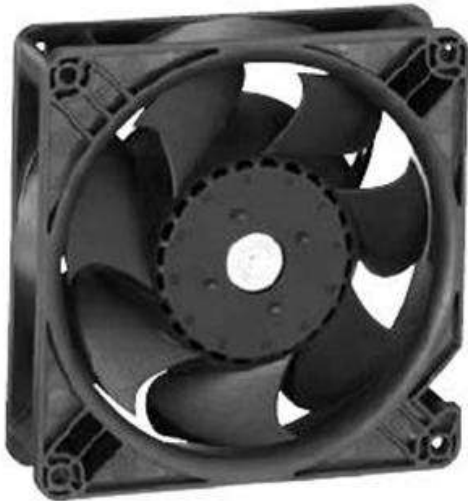

Figure 1. A "Ebmpapst” DC fan. 
voltage and $3.3 \mathrm{~V}$ voltage adjustment chip LM1117. The $28 \mathrm{~V}, 5 \mathrm{~V}$ and $3.3 \mathrm{~V}$ have met the system power supply requirements. The supply voltage of the protection circuit and the current sampling circuit is $5 \mathrm{~V}$, and the supply voltage of the microprocessor circuit and the storage circuit is $3.3 \mathrm{~V}$.

\subsection{Microprocessor Circuit}

The core of the cooling protection circuit board adopts the highest-grade series PIC18F97J60 of MICROCHIP 8-bit MCU. This MCU has $1 \mathrm{Mb}$ flash memory, built-in 10 base-T port Ethernet module, 5 timer modules, 4 external interrupt pins, 10 bits 16 Channel ADC, etc. Since the cooling circuit uses the network interface to output the speed and total current data of the three fans, the network interface function is very important. The microprocessor circuit contains an external $256 \mathrm{~Kb}$ EEPROM memory circuit that communicates with the microprocessor using the SPI bus (Figure 2). The memory is used to store network system information such as MAC address, IP address and embedded web page information.

\subsection{Protection Detection Input and Current Sampling Circuit}

Since the fan protection output is an OC open circuit output, the protection detection uses an optocoupler isolation output to the external interrupt terminal of the MCU, and the protection output is actually the fan speed pulse output. Since this microcontroller has four external interrupts, it fully satisfies the three measurement ends without any expansion. The external interrupt is triggered by a falling edge, so the frequency of each port can be measured completely. The

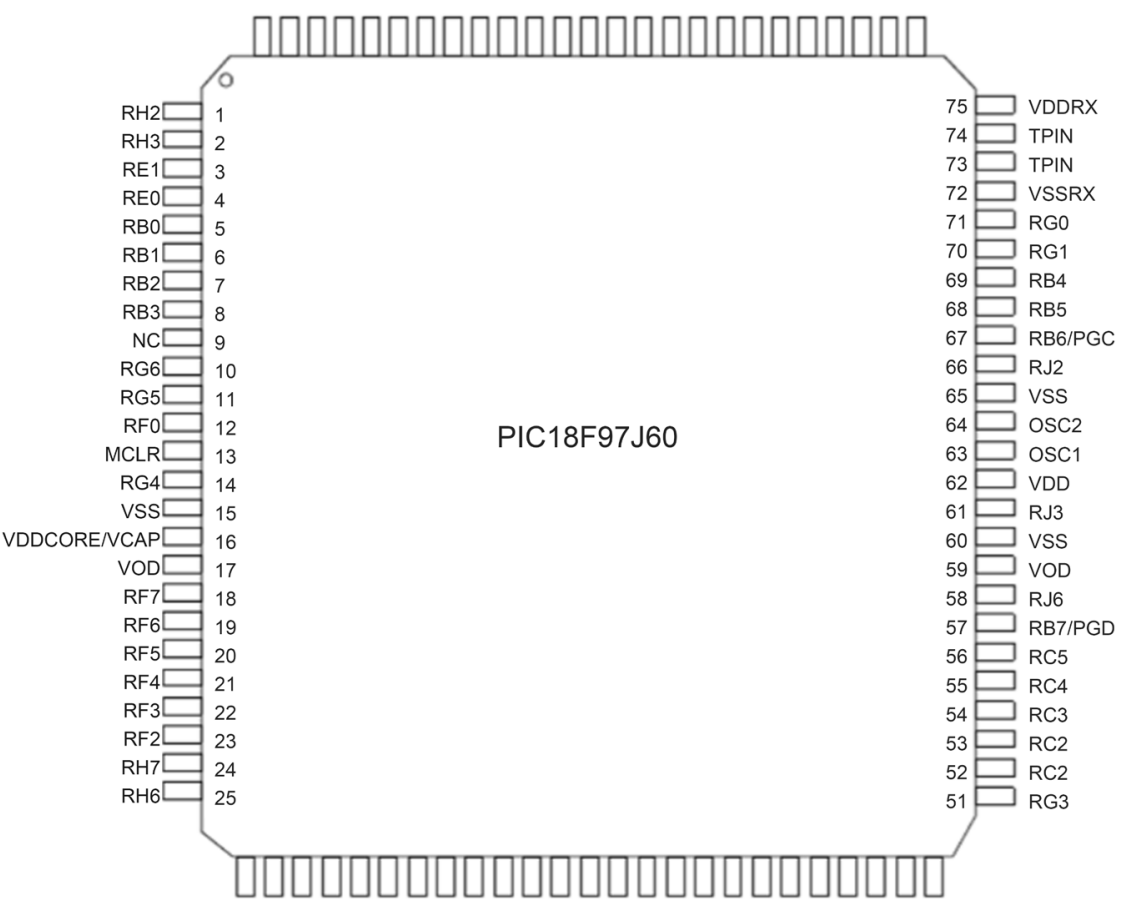

Figure 2. PIC18F97J60PIN diagram. 
current sampling circuit adopts Kang manganese resistance, and the temperature coefficient of Kang manganese resistance is small. After being amplified by the operational amplifier, it is sent to the AN0 channel of the single chip for measurement (Figure 3(a)).

\subsection{Protection Output and Network Output Circuit}

When the MCU detects that the fan speed is lower than the built-in protection value of the software or the current is significantly lower than the normal value, the output level is high, the optocoupler is turned on, and the optocoupler output terminal is directly connected with the transmitter klystron wind flow, due to the quick adjustment. The pipe flow is also alarmed when it is turned on. Therefore, the quick-regulating pipe flow output and the modulator air-cooling circuit are combined with the circuit. When any one has an alarm, a wind flow alarm is generated, so that the transmitter automatically disconnects the high voltage, effectively protect the transmitter modulator. The network interface output is relatively simple, using HR91105A network interface, built-in isolation interface and network LED interface indication (Figure 3(b)).

\section{Software Design}

Since the cooling protection board circuit has a built-in simple embedded WEB page, this webpage can display the current current speed and total current of the three fans and dynamically modify the Ethernet IP, subnet mask and gateway address (Figure 4). Therefore, the software design is divided into two parts of the single-chip system operating software and embedded web software. The system external interrupt is set to a high priority interrupt, and the software counter accumulates the pulses as each external interrupt arrives. The MCU system software has a global $1 \mathrm{Ms}$ TICK timer built in, which can be accumulated indefinitely for several decades. Each task in the system can be detected in $1 \mathrm{Ms}$. The

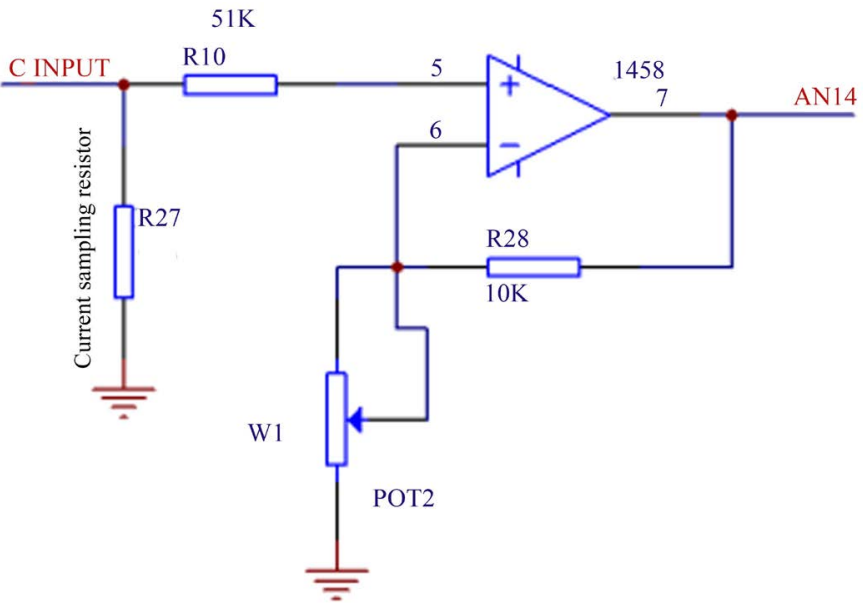

(a)

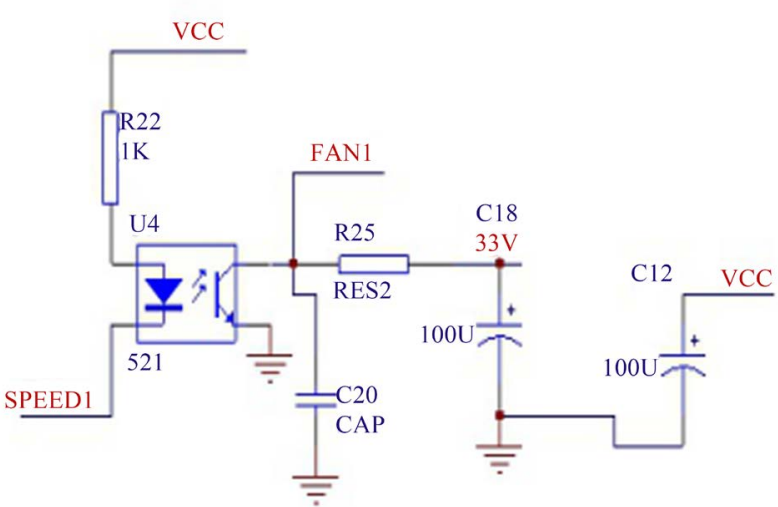

(b)

Figure 3. (a) Current sampling circuit (b) Fan wind speed measurement input circuit. 


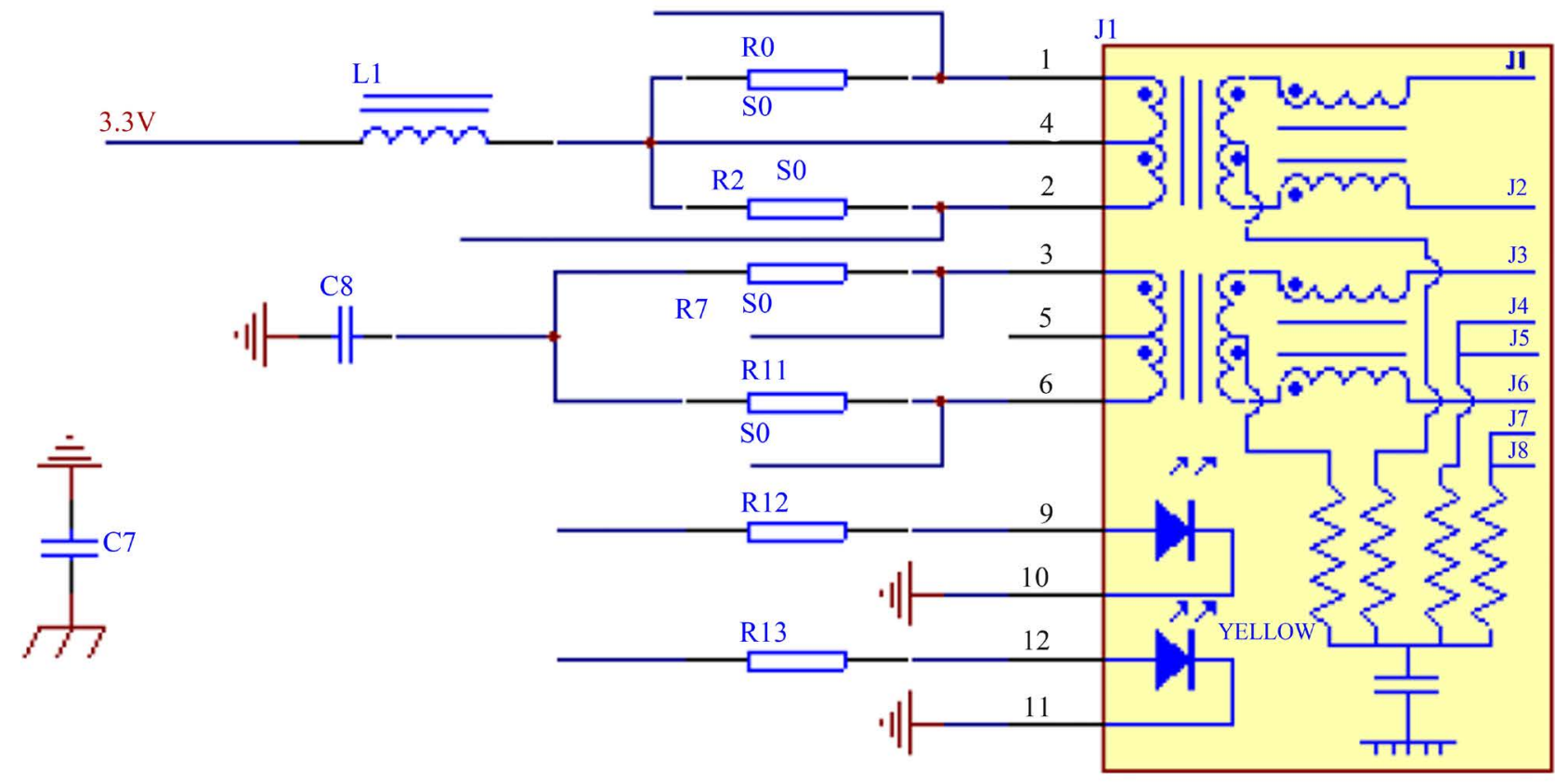

Figure 4. Network interface output.

wind speed measurement of the fan uses the $1 \mathrm{Ms}$ drip timer to form the $1 \mathrm{~S}$ frequency. Measurement: When the $1 \mathrm{~S}$ timing arrives accurately, the number of three external interrupts is read out for conversion output, and the software counter is cleared. Any item in the network system can be processed in 1 Ms (Figure 5).

The embedded web software is based on the TCP/IP protocol stack provided by MICROCHIP. The embedded webpage is written in HTML language. Since the webpage is stored in the external memory, its size is $256 \mathrm{~Kb}$, and the processor is a small system. The webpage can only work statically, and the image can only be small. After the webpage is processed, the MPFS2 tool is used to convert the webpage into data storage to the external EEPROM memory. Also mark the various dynamic variables used by the web page and the index in HTTPRINT.H. The CustomsHttpApp.C file is provided in the TCP/IP stack, describing the use of dynamic variables and the use of dynamic variables for software association with fan speed and current. Complete the web design, CustomsHttpApp.C and HTTPRINT.H file creation, it can realize the embedded webpage of the micro system.

\section{Conclusion and Discussion}

After the cooling system is designed and installed in the radar station in Xi'an, China and other places, after long-term operation, the comprehensive test shows: the system runs well, the fan parameters are clear at a glance, and the functions of the system are successfully realized, which reduces the probability of major failure of the transmitter. It effectively improves the operational level of the radar system. The cooling system described in this article has been installed 


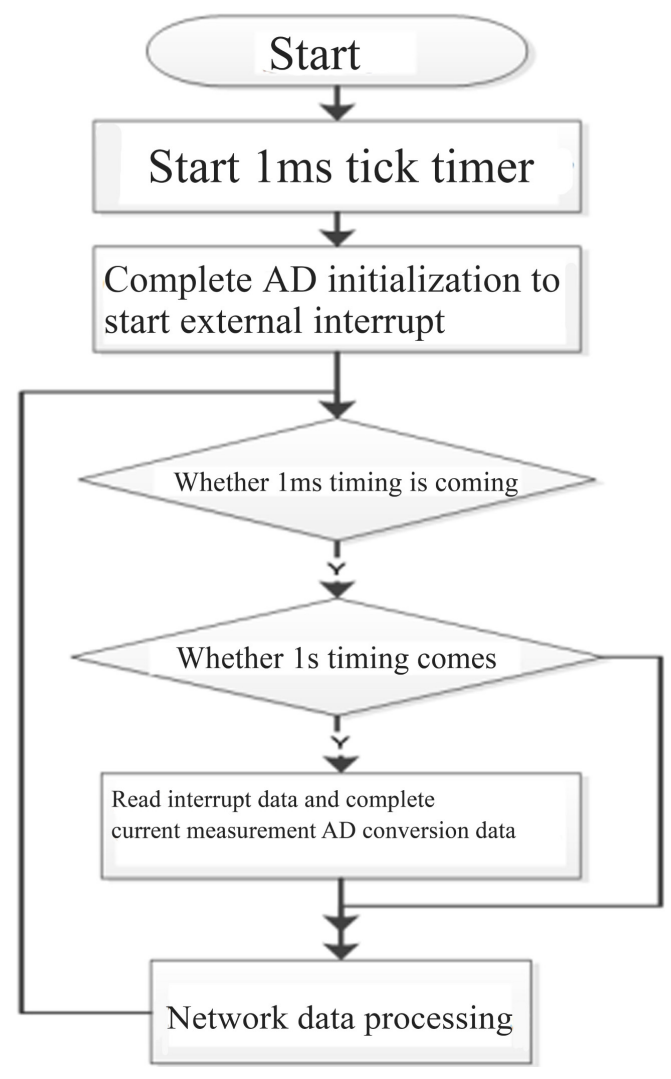

Figure 5. Software data flow chart.

on some of China's weather radars and is used by various user units. Since weather radars are very important for China's weather forecast, they can only be used for operations and cannot be used for scientific research. It is impossible for each user unit to conduct tests and obtain data in accordance with a certain standard. However, the feedback from each user unit to the system is good.

As a key device of radar, the transmitter has high power density and requires good heat dissipation. With the development of modern radar technology and the continuous advancement of power device manufacturing technology, the assembly density and power density of radar transmitters continue to increase. The feasible cooling method and excellent cooling effect have become the increasingly important support for the entire radar with high reliability indicators. The effect of temperature on the performance of the radar system is important. Too high or too low temperature will cause electronic components to malfunction, which in turn will cause the entire radar equipment to fail. The effect of temperature on the performance of the device is more prominent in high-power radar transmitters.

The optimization of radar transmitter thermal design should be synchronized with circuit design and thermal structure design, thermal simulation and experimental complementation, and integrated cooling of system and components to ensure good heat dissipation and specified operating temperature of modern radar. 


\section{Acknowledgements}

The research was funded by the National High Technology Research and Development Program 863 Project of China (2007AA061901), the National Natural Science Foundation of China (40775029), and the Liaoning Provincial Radar Maintenance and Maintenance Platform Project, China.

\section{Conflicts of Interest}

The authors declare no conflicts of interest regarding the publication of this paper.

\section{References}

Ahmadpoor, N., \& Farshidi, E. (2012). A New Approach to Complex Bandpass Sigma Delta Modulator Design for GPS/Galileo Receiver. Circuits and Systems, 3, 35-41. https://doi.org/10.4236/cs.2012.31006

Casano, G., \& Piva, S. (2015). Air-Water Cooling System for Switch-Mode Power Supplies. Journal of Electronics Cooling and Thermal Control, 5, 66-75. https://doi.org/10.4236/jectc.2015.53005

Dhiram, K., \& Wang, Z. H. (2016). Evaluation on Radar Reflectivity-Rainfall Rate (Z-R) Relationships for Guyana. Atmospheric and Climate Sciences, 6, 489-499. https://doi.org/10.4236/acs.2016.64039

Huang, J.-F., Lai, Y.-C., Lai, W.-C., \& Liu, R.-Y. (2011). Chip Design of a Low-Voltage Wideband Continuous-Time Sigma-Delta Modulator with DWA Technology for WiMAX Applications. Circuits and Systems, 2, 201-209. https://doi.org/10.4236/cs.2011.23029

Naphon, P., \& Wongwises, S. (2011). Experimental Study of Jet Nanofluids Impingement System for Cooling Computer Processing Unit. Journal of Electronics Cooling and Thermal Control, 1, 38-44. https://doi.org/10.4236/jectc.2011.13005

Rihan, F. A., \& Collier, C. G. (2010). A Basis for Improving Numerical Forecasting in the Gulf Area by Assimilating Doppler Radar Radial Winds. International Journal of Geosciences, 1, 70-78. https://file.scirp.org/Html/2388.html https://doi.org/10.4236/ijg.2010.12010

Sayama S., \& Ishii, S. (2013). Suppression of Log-Normal Distributed Weather Clutter Observed by an S-Band Radar. Wireless Engineering and Technology, 4, 125-133. https://doi.org/10.4236/wet.2013.43019

Yeo, J., Yamashita, S., Hayashida, M., \& Koyama, S. (2014). A Loop Thermosyphon Type Cooling System for High Heat Flux. Journal of Electronics Cooling and Thermal Control, 4, 128-137. https://doi.org/10.4236/jectc.2014.44014

Zheng, Z., Chen, Y., \& Cao, H. (2018). Application Analysis of the Emergency Communication System in Weather Radar. Journal of Geoscience and Environment Protection, 6, 167-174. https://doi.org/10.4236/gep.2018.68012 